\title{
Chondrocyte Differentiation is Modulated by Frequency and Duration of Cyclic Compressive Loading
}

\author{
Steven H. Elder, ${ }^{1,5}$ Steven A. Goldstein, ${ }^{2}$ James H. Kimura, ${ }^{3}$ Louis J. Soslowsky, ${ }^{4}$ \\ and DAN M. SPENGLER ${ }^{1}$ \\ ${ }^{1}$ Department of Orthopaedics and Rehabilitation, Orthopaedic Research Laboratory, Vanderbilt University, Nashville, TN, \\ ${ }^{2}$ Orthopaedic Research Laboratories, University of Michigan, Ann Arbor, MI, \\ ${ }^{3}$ Bone and Joint Center, Henry Ford Hospital, Detroit, MI, \\ ${ }^{4}$ Orthopaedic Research Laboratory, University of Pennsylvania, Philadelphia, PA, and \\ ${ }^{5}$ Department of Agricultural and Biological Engineering, Mississippi State University, Mississippi State, MS
}

(Received 31 May 2000; accepted 6 April 2001)

\begin{abstract}
As part of a program of research aimed at determining the role of mechanical forces in connective tissue differentiation, we have developed a model for investigating the effects of dynamic compressive loading on chondrocyte differentiation in vitro. In the current study, we examined the influence of cyclic compressive loading of chick limb bud mesenchymal cells to a constant peak stress of $9.25 \mathrm{kPa}$ during each of the first 3 days in culture. Cells embedded in agarose gel were subjected to uniaxial, cyclic compression at $0.03,0.15$, or $0.33 \mathrm{~Hz}$ for $2 \mathrm{~h}$. In addition, load durations of 12,54 , or 120 min were evaluated while holding frequency constant at 0.33 $\mathrm{Hz}$. For a $2 \mathrm{~h}$ duration, there was no response to loading at $0.03 \mathrm{~Hz}$. A significant increase in chondrocyte differentiation was associated with loading at $0.15 \mathrm{~Hz}$, and an even greater increase with loading at $0.33 \mathrm{~Hz}$. Holding frequency constant at $0.33 \mathrm{~Hz}$, a loading duration of 12 min elicited no response, whereas chondrocyte differentiation was enhanced by loading for either 54 or $120 \mathrm{~min}$. Although not statistically significant from the $120 \mathrm{~min}$ response, average cartilage nodule density and glycosaminoglycan synthesis rate were highest in the 54 min duration group. This result suggests that cells may be sensitive to the level of cumulative (nonrecoverable) compressive strain, as well as to the dynamic strain history.
\end{abstract}

Keywords-Cartilage, Phenotype, Compression, Chick, Limb bud. (C) 2001 Biomedical Engineering Society.

[DOI: $10.1114 / 1.1376696$ ]

\section{INTRODUCTION}

The success of methods to repair articular cartilage defects depends on identification and characterization of the physiological factors that can influence chondrocyte differentiation. It is becoming increasingly evident that one of these factors is the mechanical environment, which not only helps regulate the biosynthesis of cartilaginous matrix by mature chondrocytes, ${ }^{1,2,10,11,13,14,19}$ but

Address correspondence to Dr. Steve Elder, Ag\&Biological Engineering, Box 9632, Mississippi State, MS 39762. Electronic mail: selder@abe.msstate.edu also affects the differentiation of mesenchymal stem cells to the chondrocyte phenotype. ${ }^{3,16}$ We have previously reported that chondrogenesis in chick limb bud cellagarose cultures is enhanced by cyclic compressive loading $^{3}$ in analogous fashion to the stimulation of glycosaminoglycan (GAG) production in dynamically compressed chondrocyte-agarose constructs ${ }^{2,11}$ and in cartilage explants. ${ }^{8,10,13,14,20}$ In addition, it has been shown that this loading-induced stimulation of GAG synthesis depends on the frequency of dynamic compression $^{7,10,11,13-15}$ and on the length of exposure to the mechanical stimulus, or load duration. ${ }^{1,8,15,19}$

In our previous work using the chick limb bud cellagarose model, ${ }^{3}$ the effects of static and cyclic pressure $(0.33 \mathrm{~Hz})$ were compared. The creep strain produced by a $2 \mathrm{~h}$ static load was very similar to the cumulative (total compressive strain not recovered upon return to minimum applied pressure) component of the applied cyclic strain. In that study, chondrocyte differentiation was stimulated by cyclic loading, but not by static loading. This finding triggered the current investigation into the effects of dynamic loading frequency on differentiation.

The loading device used in the prior and current investigations applies a constant peak pressure. Because the cell-agarose constructs are viscoelastic, cumulative strain increases with time. Therefore, the current study also includes an investigation of the influence of loading duration in addition to that of frequency. Bachrach et al. ${ }^{1}$ showed that proteoglycan synthesis by bovine articular chondrocytes was stimulated by a compressive load of $0.1 \mathrm{MPa}$ applied for $10 \mathrm{~min}$, but suppressed by the same load applied for $20 \mathrm{~h}$. It was speculated that this suppression might be attributed to the increased cell deformation associated with extended load application. Such a theory is supported by the work of Wong et al. ${ }^{21}$ who demonstrated that biosynthetic activity in bovine articular cartilage explants decreased roughly linearly with the 
amount of deformation produced by unconfined, uniaxial static compression.

Based on these previous observations, we hypothesized that the effects of cyclic compression on chondrocyte differentiation would also be frequency and duration dependent. This study examines the effect of $0.03,0.15$, and $0.33 \mathrm{~Hz}$ loading frequencies and 12,54, and 120 min loading durations on GAG synthesis and cartilage nodule formation in three-dimensional agarose cultures of chick limb bud cells. The upper loading frequency was chosen to be the same as that previously shown to stimulate differentiation in this model ${ }^{3}$ and is within the range of frequencies shown to increase chondrocyte biosynthesis. ${ }^{2,14}$ The loading device was unsuitable for application of significantly higher frequencies. The longest load duration was likewise selected to be the same as that already shown to stimulate differentiation in this model. Longer durations were avoided due to the increased risk for agarose gel rupture with increasing cumulative strain.

\section{METHODS}

\section{Culture}

In compliance with U.S. federal guidelines regarding the care and use of laboratory animals, limb bud cells from stage ${ }^{5}$ 23/24 White Leghorn chick embryos were isolated and seeded into agarose as described previously. ${ }^{3}$ Briefly, limb buds were digested for $45 \mathrm{~min}$ at $37{ }^{\circ} \mathrm{C}$ in $0.025 \%$ trypsin-EDTA $/ 0.1 \%$ collagenase, pelleted by centrifugation, and resuspended at a density of 6-8 $\times 10^{6}$ cells ml ${ }^{-1}$ in Dulbecco's Modified Eagle Medium: Nutrient Mixture F-12 (D-MEM/F-12) 1:1 plus $2 \%$ fetal bovine serum (FBS). The cells from $24 \mathrm{em}-$ bryos were pooled together to establish cultures for the frequency and duration experiments, respectively. The cell suspension was mixed with an equal volume of $6 \%$ molten, low $T_{M}$ agarose (SeaPlaque, FMC Bioproducts, Rockland, ME) and cast into a slab of uniform $2 \mathrm{~mm}$ thickness. Cylindrical plugs, $11 \mathrm{~mm}$ in diameter $\times 2 \mathrm{~mm}$ thick, were cut from the slab, placed seven to a dish in specially designed stainless steel culture dishes, and immersed in $2 \mathrm{ml} \mathrm{D-MEM/F-12}$ with $2 \%$ FBS per $10^{6}$ cells. Cultures were maintained at $37{ }^{\circ} \mathrm{C}$ in a humidified atmosphere of $5 \% \mathrm{CO}_{2}: 95 \%$ air.

\section{Loading and Experimental Design}

Unconfined, uniaxial compressive loads were applied to the agarose plugs via a custom electropneumatic apparatus, the details of which have been published previously. ${ }^{3}$ The device utilizes an electropneumatic regulator to modulate a constant pressure source in response to a user-defined voltage command signal. The output pressure drives a pneumatic cylinder coupled to a smooth, stainless steel plunger, which applies an evenly distributed force to each of the unconstrained agarose plugs. For the experiments described herein, the command signal was a sine wave with a frequency of $0.03-$ $0.33 \mathrm{~Hz}$. In all cases, plugs were loaded to a constant maximum stress of $9.25 \mathrm{kPa}$ and constant minimum stress of $0.25 \mathrm{kPa}$, as determined by a pressure feedback voltage signal from the electropneumatic regulator. The resulting displacements were monitored by a LVDT mounted in parallel with the plunger.

Frequency and duration studies were carried out independently. In both studies, each experimental loading group was subjected to one loading session per day for each of the first 3 days in culture. The first loading session was started approximately $12 \mathrm{~h}$ after seeding cells into agarose. For the frequency investigation, 28 agarose plugs were divided evenly into four groups, designated by the applied frequency of loading: $0 \mathrm{~Hz}$ (control), $0.03 \mathrm{~Hz}, 0.15 \mathrm{~Hz}$, and $0.33 \mathrm{~Hz}$. The duration of loading was held constant at $2 \mathrm{~h}$ for each experimental group. An additional 28 plugs were similarly assigned to four groups designated by the duration of cyclic compressive loading: $0 \mathrm{~min}$ (control), $12 \mathrm{~min}, 54 \mathrm{~min}$, and $120 \mathrm{~min}$. In this investigation, the loading frequency was fixed at $0.33 \mathrm{~Hz}$. Plugs in the control groups were not loaded.

\section{Assays of Chondrogenesis}

In both studies, chondrogenesis was assessed on day 8 , following 5 days of static culture. The extent of chondrocyte differentiation was judged from the rate of GAG synthesis, as indicated by the rate of $\left[{ }^{35} \mathrm{~S}\right]$ sulfate incorporation into macromolecules, and from the density of cartilage nodules on histological sections. All agarose disks were halved diametrically, and half of each disk was transferred to a 24-well culture plate. Each hemidisk was incubated for $4 \mathrm{~h}$ with $1 \mathrm{ml}$ of D-MEM/F-12 and $2 \%$ FBS containing $20 \mu \mathrm{Ci}\left[{ }^{35} \mathrm{~S}\right]$ sulfate (NEX-041, DuPont NEN, Boston, MA). Following removal of the radioactive medium, disks were incubated for $12 \mathrm{~h}$ at $60{ }^{\circ} \mathrm{C}$ in a $50 \mathrm{mM}$ sodium acetate solution $(\mathrm{pH}$ 6.0) containing $280 \mu \mathrm{g} \mathrm{ml}^{-1}$ papain. The digestate was desalted on PD-10 columns (Pharmacia Biotech, Piscataway, NJ) that had been equilibrated in sodium acetate buffer, and incorporation of ${ }^{35} \mathrm{~S}$ into the macromolecular fraction was determined by liquid scintillation counting.

The other half of each agarose plug was fixed in either ruthenium hexammine trichloride ${ }^{6}$ or $10 \%$ neutral buffered formalin, and embedded in plastic (Immunobed, Polysciences, Warrington, PA). Thick $(7 \mu \mathrm{m})$ sections were stained with toluidine blue and viewed with a standard light microscope at $60 \times$ magnification. Cartilage nodules (identified by large rounded cells surrounded by metachromatically staining extracellular matrix) were 
counted within a defined area on multiple microscopic fields from triplicate sections of each agarose disk, thereby allowing the number of nodules to be normalized by the total area (density of nodules $=$ total number of nodules $\times$ total area $^{-1}$ ).

On some sections, differentiation to cartilage was confirmed using immunohistochemistry to detect the presence of type II collagen. After rehydration, these sections were incubated overnight at $37^{\circ} \mathrm{C}$ with a primary mouse antibody to chick type II collagen (II-II6B3, Development Studies Hybridoma Bank, Iowa City, IA), washed extensively in phosphate buffered saline (PBS), and then incubated for $3 \mathrm{~h}$ at $37^{\circ} \mathrm{C}$ with fluorescein isothiocyanate-conjugated anti-mouse $\mathrm{IgG}$. Following more washing in PBS, the sections were dehydrated, cleared in xylenes, and mounted with Prolong ${ }^{\mathrm{TM}}$ Antifade (Molecular Probes, Eugene, OR). On negative control sections, PBS was substituted for the primary antibody. Sections were viewed on a Leica TCSNT Confocal laser scanning microscope (Mississippi State Electron Microscope Center).

\section{Statistics}

Statistically significant differences among treatment groups were detected by Kruskal-Wallis one-way analysis of variance and test for multiple comparisons at the 95\% confidence level. Nonparametric statistics were chosen because of the relatively small sample sizes.

\section{RESULTS}

\section{Frequency}

Due to the viscoelastic nature of the agarose, loading in pressure-control mode produced two components of strain: a dynamic, peak-to-peak $(p-p)$ strain plus a monotonically increasing, cumulative compressive strain. Peak-to-peak strain is defined as the difference between the maximum and minimum strains applied during one loading cycle. The frequency of this cyclic strain component followed that of the applied pressure, but the two wave forms were slightly out of phase (pressure was monitored somewhat upstream of strain, with several feet of pneumatic tubing separating the transducers). During loading, cumulative displacement was that portion of the starting thickness $(2 \mathrm{~mm})$ not recovered upon return to the minimum applied stress. Thus cumulative strain (analogous to creep strain under static loading conditions) was defined as cumulative displacement divided by starting thickness. When duration was held constant at $2 \mathrm{~h}$ and the frequency varied, the applied stress produced approximately the same peak-to-peak axial disk strain in all three experimental groups: $\sim 10 \%(0.2 \mathrm{~mm})$ initially,
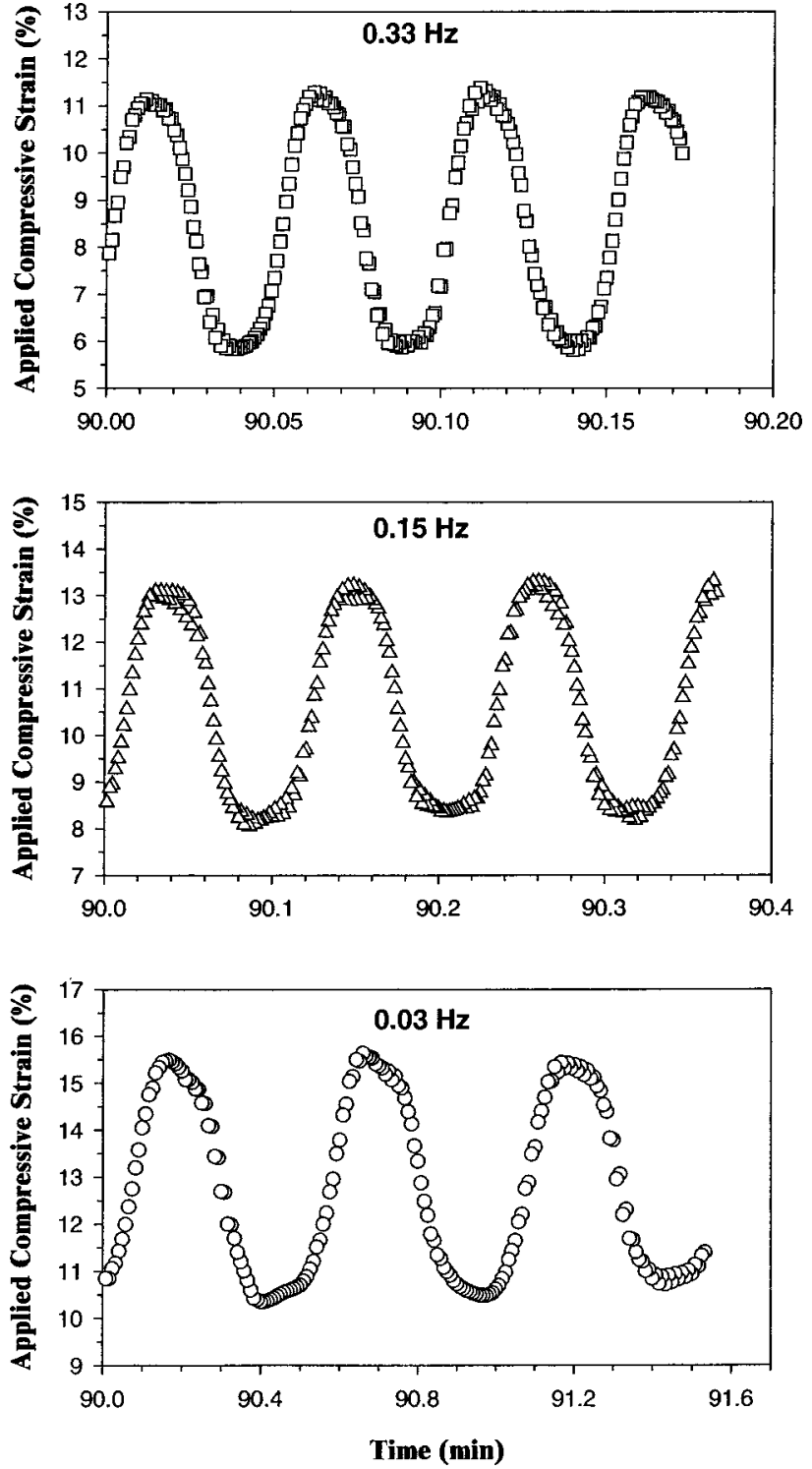

FIGURE 1. Applied compressive strain waveforms captured at the 90 min point of separate 120 min loading sessions. The horizontal axis for each graph is Time in units of minutes.

tapering to $\sim 5 \%(0.1 \mathrm{~mm})$ by the end of the loading session. This pattern was consistent over all three loading sessions.

Examples of applied compressive strain waveforms at the 90 min point during a loading session are shown in Fig. 1. The device for applying compressive loads to the cell-agarose constructs is not a closed-loop system, and there are nonlinearities in the applied strain waveforms. Harmonic distortion of the applied strain waveforms was analyzed using LabView software (National Instruments, Austin, TX). This analysis revealed that the power in the $n$th upper harmonic frequency compared to that of the fundamental (applied loading) frequency was 0.113 


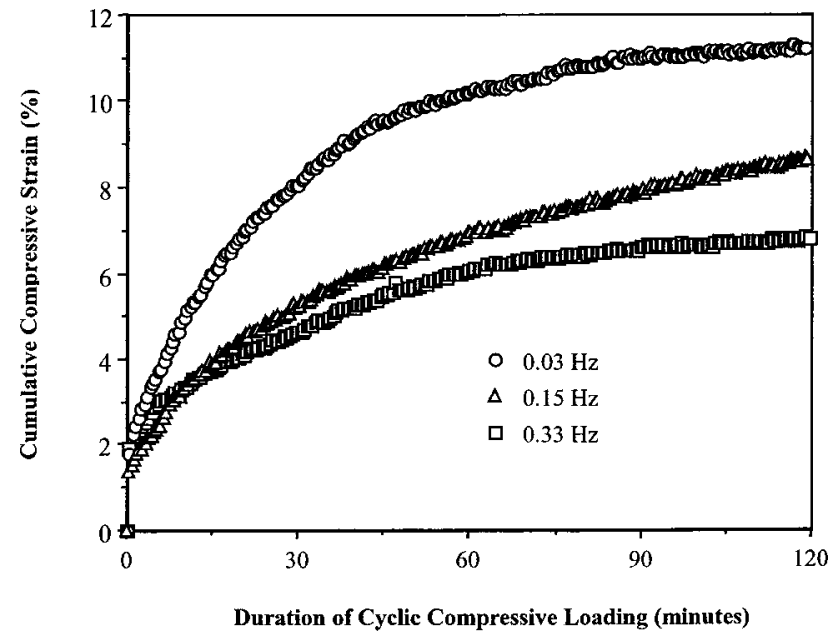

FIGURE 2. Cumulative strain histories from typical $2 \mathrm{~h}$ loading sessions.

$\pm 0.012,0.016 \pm 0.009$, and $0.034 \pm 0.020$ for $n=1,2,3$ for all three test frequencies. Never did the power in a higher harmonic frequency exceed $13 \%$ of the power in the fundamental frequency and was typically much lower.

Magnitude of the cumulative strain varied according to the loading frequency; a higher loading frequency was associated with a greater cycle-to-cycle recovery of disk thickness, and therefore a lower cumulative strain (Fig. 2 ). By the end of the $2 \mathrm{~h}$ loading session, cumulative axial strain of disks in the $0.03 \mathrm{~Hz}$ group was almost twice that of disks in the $0.33 \mathrm{~Hz}$ group. The total number of loading cycles was also a function of loading frequency. Disks in the $0.03,0.15$, and $0.33 \mathrm{~Hz}$ groups were subjected to 240,1080 , and 2400 cycles, respectively, during each loading session.

As shown in Fig. 3, there was a roughly linear rela-

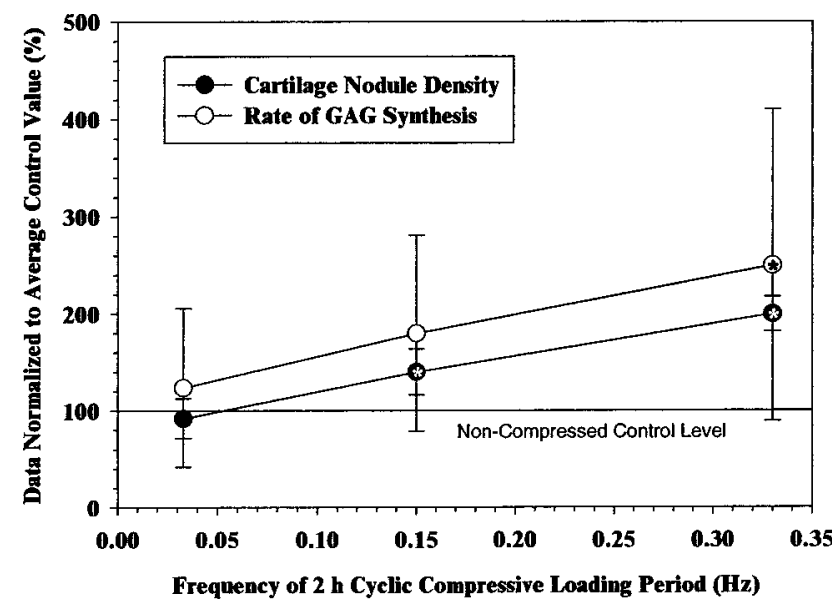

FIGURE 3. Effect of cyclic compressive loading frequency on chondrogenesis (assayed on day 8). Asterisks indicate statistically significant difference from control level (100\%). tionship between chondrogenesis and loading frequency. While loading at $0.03 \mathrm{~Hz}$ had almost no effect on the density of cartilage nodules relative to controls, loading at $0.15 \mathrm{~Hz}$ significantly increased nodule density by $50 \%$. Furthermore, loading at $0.33 \mathrm{~Hz}$ enhanced cartilage nodule density by an additional $40 \%$ over loading at 0.15 Hz. Both these differences were statistically significant $(p<0.05)$. Although the same trend was reflected in the rate of GAG synthesis, there were fewer statistically significant differences among groups due to the large intragroup variability. With respect to the rate of GAG synthesis, the only significant difference was between the $0.33 \mathrm{~Hz}$ group and controls. Cartilage nodules were unevenly distributed throughout the agarose disks; there were fewer in the center of the disks and more at the periphery. However, there were no apparent differences in the spatial distribution of nodules among the control and experimental groups. Although not quantified in this study, the distributions appeared qualitatively similar to those observed previously in the same model. ${ }^{3}$

For the indirect immunofluorescent assay, positive staining was associated with cells in presumptive cartilage nodules, indicating the presence of type II collagen synthesized by these cells (Fig. 4). Type II collagen was not detected in or around cells that did not resemble chondrocytes morphologically and were not surrounded by extracellular matrix that exhibited metachromasia with toluidine blue. There was no apparent difference in fluorescence intensity among chondrocytes in the experimental and control groups.

\section{Duration}

Axial strain of the agarose disks in all duration study groups followed the same pattern as that shown in Fig. 2 for the $0.33 \mathrm{~Hz}$ series. Thus, disks in the 12 min group were subjected only to $p-p$ strains at the high end of the range, or approximately $7 \%$, and a rapidly increasing cumulative strain that never exceeded $4 \%$. Between 12 and 54 min, $p$ - $p$ strain steadily decreased into the $5 \%-6 \%$ range, while the rate of increase in cumulative strain slowed, approaching $6 \%$ toward the end of each session. Those disks in the 120 min group were subjected to an additional period of near steady-state loading at $\sim 5 \% p-p$ strain superimposed on a very slowly increasing cumulative strain of $\sim 6 \%$.

While loading for $12 \mathrm{~min}$ ( 240 cycles) did not significantly affect chondrogenesis, loading for either $54 \mathrm{~min}$ (1080 cycles) or $120 \mathrm{~min}$ (2400 cycles) significantly stimulated differentiation (Fig. 5). GAG synthesis was elevated $\sim 80 \%$ by 54 min loading and $\sim 50 \%$ by 120 min loading relative to controls $(p<0.05)$. Similarly, nodule density was significantly increased about twofold in both cases $(p<0.05)$. The differences between the 54 and 120 min groups were not statistically significant. 

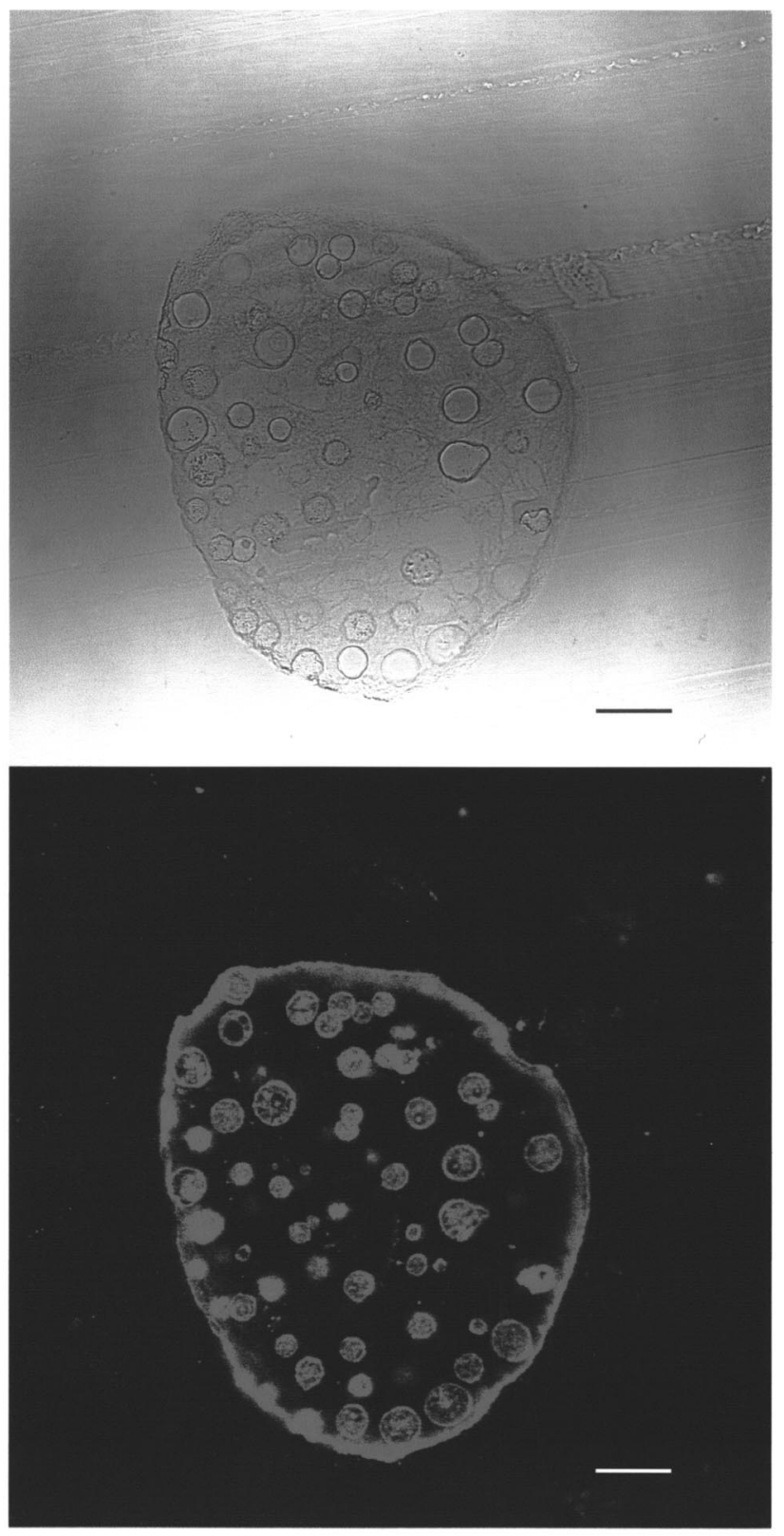

FIGURE 4. Demonstration of indirect immunofluorescent staining of type II collagen in a presumptive cartilage nodule. Top and bottom images are of the same microscopic field viewed under transmitted light and via confocal laser scanning, respectively: bar $=\mathbf{5 0} \mu \mathrm{m}$.

It should be noted that direct comparisons between groups in the frequency and duration studies cannot be made because the cells originated from separate batches of embryos, and slight variations in the stage of the embryos, the extent of cell dissociation, and the overall cell density can influence chondrogenesis and perhaps modulate the effects of loading. In the authors' experience to date, all trends in the results reported here are the same across different batches of embryos.

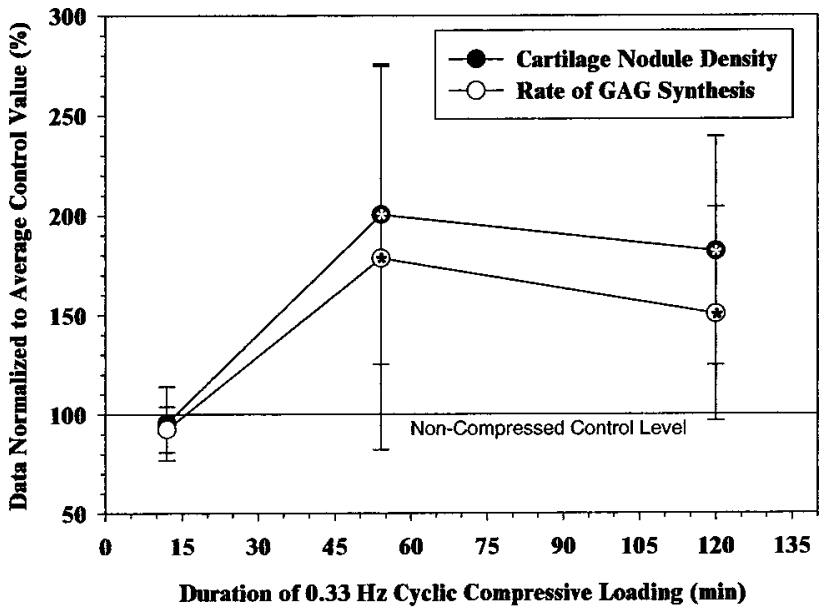

FIGURE 5. Effect of cyclic compression duration on chondrogenesis (day 8). Asterisks indicate statistically significant difference from control level (100\%).

\section{DISCUSSION}

Recent studies have clearly demonstrated that mechanical signals have the potential to regulate chondrocyte differentiation. ${ }^{3,16}$ We believe that understanding the details of this regulation can ultimately improve the outcome of attempts to repair and regenerate articular cartilage. Therefore, the overall objectives of this research are to characterize the mechanical loads that influence chondrocyte differentiation and to identify the biological pathways involved. We have previously reported that chondrogenesis was differentially affected by $2 \mathrm{~h}$ dynamic and static compressive loading, with dynamic loading ushering significantly more cells into the chondrogenic pathway. In this model, evidence of differentiation of the limb bud mesenchymal cells to the chondrocyte phenotype comes from several sources: a change in cell morphology, an accumulation of extracellular matrix exhibiting metachromasia with toluidine blue, a sharp increase in ${ }^{35} \mathrm{~S}$ incorporation, and the immunohistochemically detected presence of type II collagen.

In the current study, we demonstrate that the cyclic loading-induced stimulation of chondrogenesis depends on loading frequency and duration. In terms of the proportion of the initial chick limb bud cell population that differentiates into chondrocytes, our results suggest that there is a negligible effect of loading at frequencies at or below $0.03 \mathrm{~Hz}$, whereas loading at $0.15-0.33 \mathrm{~Hz}$ is stimulatory in a dose-dependent manner. Although the applied strain waveforms were not pure sinusoids, power spectrum density analysis indicated that it is unlikely that the observed variations in differentiation could be attributed to anything other than the differences in the fundamental applied loading frequency. The applied strain had both a dynamic and monotonically increasing (cumulative) component, which is similar to the strain produced 
by static loading at constant stress. The dynamic component was more pronounced at higher frequencies where the agarose behaves more elastically. Our earlier study suggests that the cumulative component of loading has a minor, possibly inhibitory, effect on chondrocyte differentiation, and that stimulation of chondrogenesis depends on dynamic strain. Thus, in the present study, it is not surprising that stimulation of chondrogenesis was diminished at lower frequencies, which were associated with greater cumulative strain. This finding is in agreement with that of Torzilli et al. ${ }^{18}$ who suggested that the decreased metabolic response of cartilage explants to a pressure-controlled, sinusoidal compression was dominated by the static component of the dynamic load.

With respect to loading frequency, the chick limb bud cells in our study displayed the same trend in differentiation as chondrocytes generally exhibit in proteoglycan synthesis: less stimulation or even inhibition at lower frequencies and greater stimulation at higher frequencies in the range of $0.1-1 \mathrm{~Hz}$. For example, Sah et al. ${ }^{14}$ reported that oscillatory compressive strains in cartilage explants of $<5 \%$ stimulated ${ }^{35} \mathrm{~S}$ incorporation at $0.01-1$ $\mathrm{Hz}$, but had no effect at $0.0001-0.001 \mathrm{~Hz}$. Similarly, Palmoski and Brandt ${ }^{13}$ and Larsson et al. ${ }^{10}$ reported suppression of proteoglycan synthesis in cartilage explants loaded at low frequency (duty cycle of $60 \mathrm{~s}$ on/60 s off), and enhanced production at high frequency $(4 \mathrm{~s}$ on/11 s off and $2 \mathrm{~s}$ on/2 s off, respectively). For chondrocytes in agarose, Buschmann et al. ${ }^{2}$ demonstrated that lowamplitude cyclic compression (6\% p-p) elevated proteoglycan synthesis at $0.01-1 \mathrm{~Hz}$, whereas Lee and Bader $^{11}$ found that a $15 \%$ dynamic compressive strain was inhibitory at $0.3 \mathrm{~Hz}$, stimulatory at $1 \mathrm{~Hz}$, and did not change proteoglycan synthesis at $3 \mathrm{~Hz}$.

One possible explanation for the dependency on loading frequency is the effect of frequency on interstitial fluid flow and hydrostatic pressure. Changing the frequency of loading alters the radial profile of hydrostatic pressure and fluid flow velocity within the agarose plug. However, in the current study the spatial distribution of cartilage nodules was not markedly altered by the frequency of loading; that is, stimulation of differentiation did not appear to be confined to either the outer ring or to the center of the disks. Another possibility is that the sensitivity to loading frequency is related to cell deformation. Compression of cell/agarose constructs has been shown to cause cell strain of approximately the same magnitude as that applied to the agarose. ${ }^{4,9}$ Furthermore, Kim et ll $^{7}$ have provided evidence that cyclic loadinginduced stimulation of chondrocyte biosynthesis at amplitudes up to $10 \%$ strain was more likely the result of altered cell shape (and/or fluid flow) than changes in hydrostatic pressure. Although by no means conclusive, our results seem to support the theory that enhanced chondrogenesis was due to the compressive loadinginduced cell deformation.

In addition to demonstrating an effect of loading frequency on chondrogenesis, our results also indicate that cells are sensitive to the load duration. Thus, cells may be sensitive to the time of exposure to cyclic compression and/or to the total number of loading cycles. The lack of chondrogenic stimulation observed in the $12 \mathrm{~min}$ loading group $(0.33 \mathrm{~Hz})$ suggests that there is a threshold number of cycles between 240 and 1080 per $24 \mathrm{~h}$ below which loading has a negligible effect, regardless of frequency. The existence of a threshold duration that must be exceeded to elicit a response is evidence of a lengthy signal transduction pathway and suggests that cells may have a mechanism for storing information about the stress/strain history.

Above the duration threshold, the response to cyclic loading may be related to the level of nonrecoverable or cumulative strain in the cell/agarose disk, which increases over time. The data suggest that the final half of each $2 \mathrm{~h}$ cyclic loading session to a constant maximum stress may have had no effect or may possibly have had an adverse effect on differentiation. Cartilage explants subjected to load-controlled static compression displayed a similar pattern of aggrecan mRNA synthesis, with increased expression at $1 \mathrm{~h}$ followed by a decline to baseline levels at 4 and $24 \mathrm{~h}^{19}{ }^{19}$ Furthermore, proteoglycan synthesis by cartilage explants under constant compressive load exhibited the same trend; a 10 min load stimulated synthesis, whereas the same load applied for $20 \mathrm{~h}$ suppressed synthesis. ${ }^{1}$ Finally, it has been shown that increasing the duration of cyclic compressive explant loading over the range of 1-6 days reduced proteoglycan synthesis. ${ }^{15}$ Thus the differentiation of chick limb bud cells in response to continuously applied cyclic compression seems to parallel the biosynthesis of mature chondrocytes, with a short term duration having a stimulatory effect and a longer duration having a reduced or inhibitory effect. As discussed above for the frequency study, the transition from one type of response to the other may be related to the increasing dominance of the cumulative component of strain as load duration increases.

In addition to furthering the characterization of chondrotropic mechanical stimuli, our results may provide insight into the biological pathways of mechanotransduction that lead to a change in gene expression. For example, the above speculation that dynamic cell strain is critical to the loading-induced stimulation of differentiation points to the cell membrane and cytoskeleton as potentially important parts of the mechanotransduction mechanism. The cytoskeletal network of microtubules substantially contributes to the cell's resistance to compression, and colchicine, a drug that disrupts microtubules, has been found to suppress chondrocyte differentiation in vitro. ${ }^{17}$ In addition, Martina et al. ${ }^{12}$ identified a 
stretch-activated potassium channel in the membrane of porcine articular chondrocytes, and Wright et al. ${ }^{22}$ have demonstrated that stretch-activated ion channels in the cell membrane are associated with chondrocyte hyperpolarization following cyclical pressure-induced strain.

In summary, cyclic loading-induced stimulation of chondrocyte differentiation from chick limb bud cellagarose cultures was influenced by loading frequency and duration. Differentiation was unaffected by loading at the lowest frequency, $0.03 \mathrm{~Hz}$, or the shortest duration, $12 \mathrm{~min}$. The greatest stimulation occurred after loading at $0.33 \mathrm{~Hz}$ for approximately $1 \mathrm{~h}$. We speculate that the existing level of cumulative or creep strain is an important factor in the response to dynamic loading.

\section{ACKNOWLEDGMENTS}

Funding was provided by NIH Grant No. AR20557, the Whitaker Foundation, the Frederick J. Fischer Endowment Fund, the Horace H. Rackham School of Graduate Studies at the University of Michigan, and the Vanderbilt University Department of Orthopaedics and Rehabilitation. The II-II6B3 monoclonal antibody developed by Dr. Thomas Linsenmayer was obtained from the Developmental Studies Hybridoma Bank developed under the auspices of the NICHD and maintained by the Department of Biological Sciences, The University of Iowa, Iowa City, IA 52242. Eggs were provided by Bilbie Aviaries, Ann Arbor, MI, and by Tyson Foods, Shelbyville, TN. The authors would also like to thank the following individuals for their technical and administrative assistance: Saul Juliao, John Dawson, Pat Commers, Debbie Hefner, and Bill Monroe.

\section{REFERENCES}

${ }^{1}$ Bachrach, N. M., W. B. Valhmu, E. Stazzone, A. Ratcliffe, W. M. Lai, and V. C. Mow. Changes in proteoglycan synthesis of chondrocytes in articular cartilage are associated with the time-dependent changes in their mechanical environment. J. Biomech. 28:1561-1569, 1995.

${ }^{2}$ Buschmann, M. D., Y. A. Gluzband, A. J. Grodzinsky, and E. B. Hunziker. Mechanical compression modulates matrix biosynthesis in chondrocyte/agarose culture. J. Cell. Sci. 108:1497-1508, 1995.

${ }^{3}$ Elder, S. H., J. H. Kimura, L. J. Soslowsky, M. Lavagnino, and S. A. Goldstein. Effects of compressive loading on chondrocyte differentiation in agarose cultures of chick limb bud cells. J. Orthop. Res. 18:78-86, 2000.

${ }^{4}$ Freeman, P. M., M. R. N. Natarajan, J. H. Kimura, and T. P. Andriacchi. Chondrocyte cells respond mechanically to compressive loads. J. Orthop. Res. 12:311-320, 1994.

${ }^{5}$ Hamburger, V., and H. Hamilton. A series of normal stages in development of the chick embryo. J. Morphol. 88:49-92, 1951.

${ }^{6}$ Hunziker, E. B., W. Herrmann, and R. K. Schenk. Improved cartilage fixation by ruthenium hexammine trichloride (RHT): A prerequisite for morphometry in growth cartilage. J. Ultrastruct. Res. 81:1-12, 1982.
${ }^{7}$ Kim, Y. J., R. L. Sah, A. J. Grodzinsky, A. H. Plaas, and J. D. Sandy. Mechanical regulation of cartilage biosynthetic behavior: Physical stimuli. Arch. Biochem. Biophys. 311:1-12, 1994.

${ }^{8}$ Kiraly, K., M. M. Hyttinen, J. J. Parkkinen, M. A. Arokoski, T. Lapvetelainen, K. Torronen, I. Kiviranta, and H. J. Helminen. Articular cartilage collagen birefringence is altered concurrent with changes in proteoglycan synthesis during dynamic in vitro loading. Anat. Rec. 251:28-36, 1998.

${ }^{9}$ Knight, M. M., D. A. Lee, and D. L. Bader. Distribution of chondrocyte deformation in compressed agarose gel using confocal microscopy. J. Cell. Eng. Incorp. Mol. Eng. 1:97102, 1996.

${ }^{10}$ Larsson, T., R. M. Aspden, and D. Heinegard. Effects of mechanical load on cartilage matrix biosynthesis in vitro. Matrix 11:388-394, 1991.

${ }^{11}$ Lee, D. A., and D. L. Bader. Compressive strains at physiological frequencies influence the metabolism of chondrocytes seeded in agarose. J. Orthop. Res. 15:181-188, 1997.

${ }^{12}$ Martina, M., J. W. Mozrzymas, and F. Vittur. Membrane stretch activates a potassium channel in pig articular chondrocytes. Biochim. Biophys. Acta 1329:205-210, 1997.

${ }^{13}$ Palmoski, M. J., and K. D. Brandt. Effects of static and cyclic compressive loading on articular cartilage plugs in vitro. Arthritis Rheum. 27:675-681, 1984.

${ }^{14}$ Sah, R. L.-Y., Y.-J. Kim, J.-Y. H. Doong, A. J. Grodzinsky, A. H. K. Plaas, and J. D. Sandy. Biosynthetic response of cartilage explants to dynamic compression. J. Orthop. Res. 7:619-636, 1989.

${ }^{15}$ Steinmeyer, J., and S. Knue. The proteoglycan metabolism of mature articular cartilage explants superimposed to continuously applied cyclic mechanical loading. Biochem. Biophys. Res. Commun. 240:216-221, 1997.

${ }^{16}$ Takahashi, I., G. H. Nuckolls, K. Takahashi, O. Tanaka, I. Semba, R. Dashner, L. Shum, and H. C. Slavkin. Compressive force promotes sox9, type II collagen and aggrecan and inhibits IL-1beta expression resulting in chondrogenesis in mouse embryonic limb bud mesenchymal cells. J. Cell. Sci. 111:2067-2076, 1998.

${ }^{17}$ Takigawa, M., T. Takano, E. Shirai, and F. Suzuki. Cytoskeleton and differentiation: Effects of cytochalasin B and colchicine on expression of the differentiated phenotype of rabbit costal chondrocytes in culture. Cell Differ. 14:197204, 1984.

${ }^{18}$ Torzilli, P. A., R. Grigiene, C. Huang, S. M. Friedman, S. B. Doty, A. L. Boskey, and G. Lust. Characterization of cartilage metabolic response to static and dynamic stress using a mechanical explant test system. J. Biomech. 30:1-9, 1997.

${ }^{19}$ Valhmu, W. B., E. J. Stazzone, N. M. Bachrach, F. SaedNejad, S. G. Fischer, V. C. Mow, and A. Ratcliffe. Loadcontrolled compression of articular cartilage induces a transient stimulation of aggrecan gene expression. Arch. Biochem. Biophys. 353:29-36, 1998.

${ }^{20}$ van Kampen, G. P., G. H. Korver, and R. J. van de Stadt. Modulation of proteoglycan composition in cultured anatomically intact joint cartilage by cyclic loads of various magnitudes. Int. J. Tissue React. 16:171-179, 1994.

${ }^{21}$ Wong, M., P. Wuethrich, M. D. Buschmann, P. Eggli, and E. Hunziker. Chondrocyte biosynthesis correlates with local tissue strain in statically compressed adult articular cartilage. $J$. Orthop. Res. 15:189-196, 1997.

${ }^{22}$ Wright, M., P. Jobanputra, C. Bavington, D. M. Salter, and G. Nuki. Effects of intermittent pressure-induced strain on the electrophysiology of cultured human chondrocytes: Evidence for the presence of stretch-activated membrane ion channels. Clin. Sci. 90:61-71, 1996. 